\title{
EVALUACIÓN DEL RECURSO HÍDRICO SUPERFICIAL EN LA SUBCUENCA HIDROGRÁFICA DEL RÍO FRÍO EN EL DEPARTAMENTO DE CUNDINAMARCA: OFERTA, DEMANDA Y CALIDAD DEL AGUA
}

\author{
EVALUATION OF THE SURFACE WATER RESOURCE IN THE \\ HYDROGRAPHIC FRÍO RIVER SUBBASIN AT CUNDINAMARCA \\ DEPARTMENT: OFFER, DEMAND AND QUALITY OF THE WATER
}

\author{
Melissa Andrea Hoyos Chaverra ${ }^{1}$ \\ Denisse Ariana Rodríguez Barrientos ${ }^{2}$ \\ Jesús Ernesto Torres Quintero ${ }^{3}$ \\ 1 Est. Ingeniería Ambiental, Universidad Libre. Colombia \\ 2 Est. Ingeniería Ambiental, Universidad Libre. Colombia \\ ${ }^{3}$ Magíster en Recursos Hidráulicos. Investigador docente Universidad Libre. Colombia \\ 1 melissaa.hoyosc@unilibrebog.edu.co \\ 2 denissea.rodriguezb@unilibrebog.edu.co \\ 3 jesuse.torresq@unilibre.edu.co
}

\section{Resumen}

Colombia es uno de los países más ricos en cuanto a recurso hídrico se refiere; sin embargo, se ha visto afectado por la alta demanda generada por las poblaciones y por el desarroIlo agroindustrial. Por ello, este estudio busca determinar si el Río Frío, como fuente de abastecimiento de agua de diversos municipios, satisface la necesidad de agua potable de éstos, estableciendo también si las actividades económicas generan un impacto negativo sobre este recurso. La evaluación se realizó en cuatro etapas: la primera consistió en la definición de la oferta hídrica por medio de la metodología de Caudal Medio Puntual establecida por el Instituto de Hidrología, Meteorología y Estudios Ambientales (IDEAM); la segunda etapa se basó en la determinación de la demanda superficial según el segundo escenario establecido por el IDEAM, teniendo en cuenta la existencia y confiabilidad de la información; la tercera etapa consistió en realizar la relación Demanda-Oferta; y la cuarta se centró en estimar la calidad del agua mediante la evaluación de los parámetros físicos y químicos, el cálculo del Índice de Calidad del Agua (ICA). Como resultado, se obtuvo que actualmente la oferta hídrica no es suficiente para satisfacer la demanda de los municipios; la cual es, 
principalmente, de índole agrario, por lo cual es necesario buscar otras fuentes de suministro de agua. Además, se encontró que la calidad del Río Frío es baja; particularmente, debido a las descargas de aguas residuales e industriales provenientes de poblaciones aledañas.

Palabras clave: calidad, demanda, oferta, subcuenca, superficial.

\section{Abstract}

Colombia is one of the richest countries in terms of water resources, however it has been affected by the high demand generated by populations and agroindustry development. Therefore, this study was focused on the determination of the Frio River capabilities, as a source of water supply for several municipalities, for satisfying their needs for drinking water, also establishing if the economic activities generate a negative impact on this resource. The evaluation was carried out in four stages: the first one consisted of the definition of the water supply by means of the Method of
Average Point Flow established by the Institute of Hydrology, Meteorology and Environmental Studies (IDEAM); the second stage was based on the determination of the surface demand according to the second scenario established by the IDEAM, taking into account the existence and reliability of the information; the third stage consisted of establishing the supply-demand relation; and the fourth one was focused on estimating the water quality by means of the evaluation of the physical and chemical parameters and the calculation of the Water Quality Index. As results, it was obtained that the current water supply is not sufficient to satisfy the demand of the municipalities, which is mainly of agrarian nature, making necessary the search of other sources for water supply, besides obtaining that quality of the Frio River is low, mainly due to the wastewater and industrial waters coming from the towns bordering it.

Keywords: demand, offer, subbasin, superficial, quality.

\section{Introducción}

Desde los comienzos de la humanidad, el agua ha jugado un papel importante en el desarrollo de la sociedad y el crecimiento de las poblaciones ha generado un incremento en el uso del agua para la producción de alimentos y de energía, entre otros, con el fin de satisfacer la demanda de los seres humanos y mejorar su bienestar. Sin embargo, los cambios en la forma en la que se usa el agua tienen efectos negativos importantes que requieren una atención urgente que garantice la sostenibilidad (UNESCO, 2006). Esto se observa en Colombia, ya que es un país rico hídricamente, en donde este recurso presenta una alta demanda, viéndose así afectado debido a los grandes asentamientos humanos y el desarrollo industrial, generando presión y escasez sobre el recurso, además de la alteración de la calidad del agua (IDEAM, 2014), siendo estas razones determinantes y necesarias para evaluar las pequeñas fuentes hídricas del país, como se realiza en este caso, al evaluar el balance oferta-demanda en el Río Frío, así como su calidad.

Debido a que el Río Frío atraviesa los municipios de Tabio, Cajicá, Chía, Cogua, Cota, Tenjo y Zipaquirá (CAR, 2006), constituye una fuente de abastecimiento para estos municipios, además de contribuir al desarrollo agrícola e industrial de toda la zona que abarca; sin embargo, estos beneficios se han visto afectados 
por el crecimiento desmedido de la población de dichos municipios y por la contaminación que se ha venido incrementando en este río como consecuencia del desarrollo industrial presente en esa zona (Venegas, 2013).

Por ende, este trabajo se centra en la determinación de la capacidad del Río Frío, como fuente de abastecimiento de agua, para satisfacer la necesidad de agua potable en los municipios mencionados anteriormente tanto para la presente como para la futura demanda en cuanto a las diversas actividades socioeconómicas en las cuales se basa la economía de los municipios aledaños. Para ello, se propone realizar una evaluación de la oferta hídrica y demanda superficial, así como de la calidad del agua mediante el análisis hidrológico con base en características morfométricas, relativas a los principales abastecimientos de agua de los diversos municipios y mediante la planificación para un manejo integral de este recurso hídrico.

\section{Materiales y Método}

Objetivo uno. Definición de la oferta hídrica de la subcuenca hidrográfica del Río Frío en Cundinamarca. Para efectos de cálculo de la oferta hídrica del Río Frío, se dispuso de la metodología de Caudal medio puntual en las corrientes de interés establecida por el Instituto de Hidrología, Meteorología y Estudios Ambientales (IDEAM,2004). La elección de este método se debe a la información disponible, dado que éste se utiliza cuando los registros de caudal son series cortas o no confiables. Este método consiste en determinar el caudal disponible de utilización de una corriente, para lo cual es necesario conocer los caudales mínimos y medios de la subcuenca, los cuales fueron obtenidos de la estación limnimétrica correspondiente. Posteriormente, con base en los caudales medios, se realizó la curva de duración de caudales, la cual permitió observar la variabilidad de los caudales en el tiempo y tener un mejor conocimiento en el manejo de la disponibilidad del agua, y por consiguiente, explicar la relación demanda-oferta. Para el cálculo del caudal de la oferta, se tomó el $50 \%$ de frecuencia de la Curva de Duración de Caudales y el caudal ecológico, el cual es el $25 \%$ del caudal mínimo mensual de la cuenca.

Los resultados aquí obtenidos se encuentran representados mediante un Sistema de Información Geográfica (SIG); específicamente, ArcGis.

Objetivo dos. Determinación de la demanda superficial en los municipios pertenecientes a la subcuenca hidrográfica del Río Frío en Cundinamarca. Para la determinación de la demanda superficial de la subcuenca hidrográfica del río Frío se dispuso de la información reportada por el Instituto de Hidrología, Meteorología y Estudios Ambientales (IDEAM,2004), el cual ofrece tres diferentes tipos de escenarios para el cálculo de la demanda según la existencia y confiabilidad de la información. En este caso, se seleccionó el escenario dos, aplicable cuando se dispone de información medida pero insuficiente; siendo las concesiones de agua de los municipios aledaños al río la información existente utilizada, la cual fue proporcionada por la Corporación Autónoma Regional de Cundinamarca (CAR). Para la información inexistente se determinó la demanda total mediante el tercer escenario propuesto por el IDEAM, implementando la ecuación (1).

\section{(1). $\mathrm{DT}=\mathrm{DD}+\mathrm{DI}+\mathrm{DA}+\mathrm{DP}+\mathrm{DS}$}

Donde:

DT: Demanda total de agua.

DD: Demanda de uso doméstico.

DI: Demanda de uso industrial.

DS: Demanda del sector de servicio.

DA: Demanda del sector agrícola. DP: Demanda del sector pecuario. 
Como se indicó en la fase uno, los resultados aquí obtenidos se encuentran representados mediante un Sistema de Información Geográfica (SIG); específicamente, ArcGis.

Objetivo tres: Relación demanda-oferta. Realizados los cálculos de oferta y demanda, se realizó una relación demanda-oferta; en primer lugar, mediante un balance; y en segundo lugar, implementando la expresión del Índice de Escasez, representada por la ecuación (2).

(2) $I e=\frac{D h}{O h} * 100 \%$

Donde:

Ie: Índice de Escasez en Porcentaje.

Dh: Demanda Hídrica Total.

Oh: Oferta Hídrica.

El resultado obtenido se evaluó a partir de la información recogida en la Tabla 1.

Tabla 1. Categorización del Índice de Escasez (IDEAM, 2004)

\begin{tabular}{l|c|c} 
Categoría & \multicolumn{1}{|c}{ Rango } & Color \\
\hline Alto & $>40 \%$ & Rojo \\
\hline Medio & $20-40 \%$ & Naranja \\
\hline Moderado & $10-20 \%$ & Amarillo \\
\hline Bajo & $<10 \%$ & Verde \\
\hline
\end{tabular}

Objetivo cuatro. Evaluación de la calidad del agua de la subcuenca hidrográfica del Río Frío en Cundinamarca. La estimación de la calidad del agua del Río Frío se realizó mediante la determinación del Índice de Calidad de Agua (ICA), metodología propuesta por el IDEAM, en ciertos tramos del Río Frío, siendo el ICA calculado a partir de la ecuación (3).

$$
I C A_{n j t}=\sum\left(W i^{*} I_{i k j t}\right)
$$

Donde:

$I C A_{n j t}$ : Índice de calidad del agua del Río Frío, en el punto de muestreo $j$ en el tiempo $t$, evaluado con base en $n$ variables.

Wi: Ponderador o peso relativo asignado a la variable de calidad i.

$I_{i k j t:}$ Valor calculado de la variable i (obtenido de aplicar la ecuación correspondiente para cada variable), en el punto de muestreo j, registrado durante la medición realizada en el semestre $\mathrm{k}$, del período de tiempo t.

n: Número de variables de calidad involucradas en el cálculo del indicador; en este caso $n$ es igual a cinco.

De modo que, el valor del ICA calculado en cada punto de muestreo se comparó con la Tabla 2 relativa a la calificación de la calidad del agua según los valores que tome el ICA.

Tabla 2. Calificación de la calidad del agua según los valores que tome el ICA (IDEAM, 2013)

\begin{tabular}{c|c}
$\begin{array}{c}\text { Categorías de valores } \\
\text { que puede tomar } \\
\text { el indicador }\end{array}$ & $\begin{array}{c}\text { Calificación } \\
\text { de la calidad } \\
\text { del agua }\end{array}$ \\
$0,00-0,25$ & Muy mala \\
\hline $0,26-0,50$ & Mala \\
\hline $0,51-0,70$ & Regular \\
\hline $0,71-0,90$ & Aceptable \\
\hline $0,91-1,00$ & Buena \\
\hline
\end{tabular}

Los resultados obtenidos de los valores del ICA para cada punto de muestreo se encuentran representados mediante un Sistema de Información Geográfica (SIG); específicamente, ArcGis. 


\section{Resultados}

Objetivo uno. Definición de la oferta hí- encontraban dentro de esta subcuenca. drica de la subcuenca hidrográfica del Río Frío en Cundinamarca. Para el análisis de los caudales medios del Río Frío se selecciona-

En la Tabla 3 se presentan las 5 estaciones seron 5 estaciones limnimétricas, las cuales se leccionadas junto con los rangos de años correspondientes y sus respectivas coordenadas.

Tabla 3. Estaciones limnimétricas de Río Frío

\begin{tabular}{l|c|c|c|c}
\hline Estación & N & E & Rango de años & Cuenca \\
\hline Pozo Hondo & 1051425 & 1001930 & $1999-2014$ & Media \\
\hline Páramo Guerrero & 1056700 & 1003500 & $1997-2014$ & Alta \\
\hline Puente Virginia & 1036500 & 1001200 & $1947-2014$ & Baja \\
\hline Puente Cacique & 1029550 & 1001330 & $2007-2014$ & Baja \\
\hline Puente Calamar & 1038840 & 999380 & $1995-2014$ & Media \\
\hline
\end{tabular}

En la Figura 1 a la Figura 5, se pueden encontrar las curvas de duración de caudales para cada una de las estaciones presentadas.

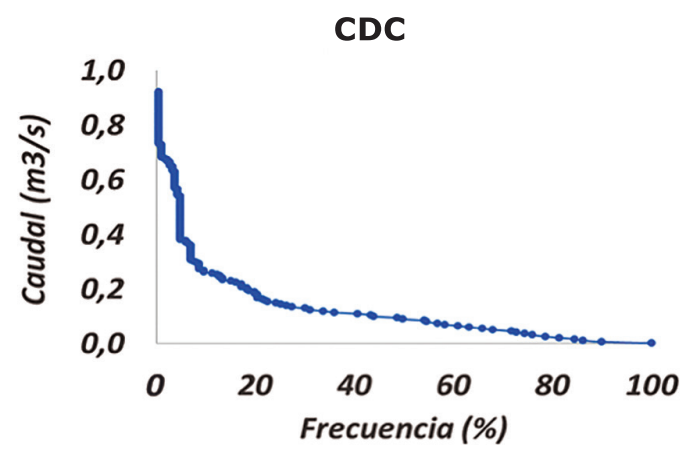

Figura 1. CDC Estación Páramo Guerrero.

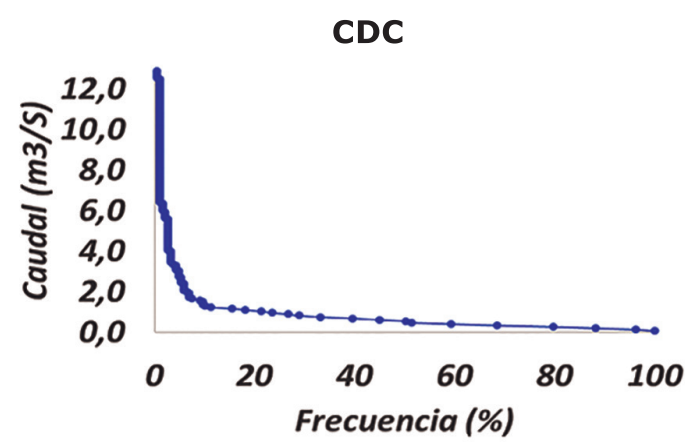

Figura 2. CDC Estación Pozo Hondo.

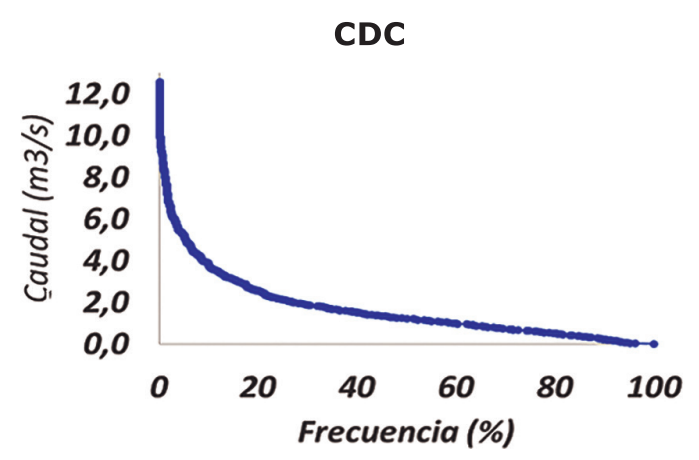

Figura 3. CDC Estación Puente Virginia.

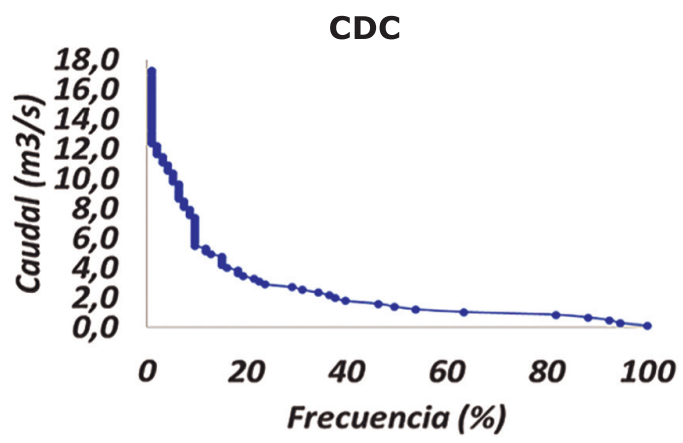

Figura 4. CDC Estación Puente Cacique. 


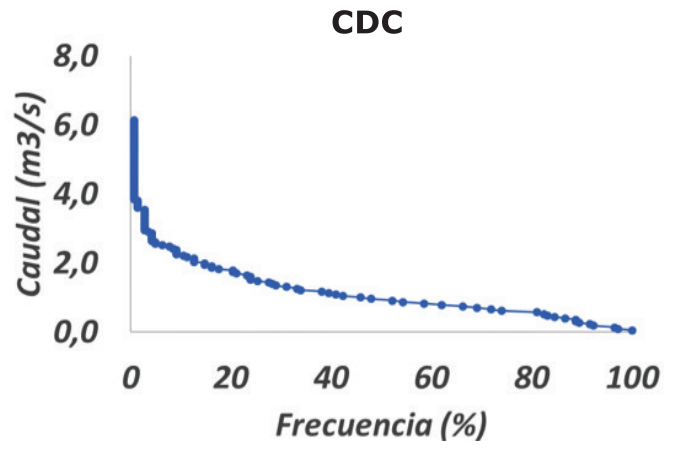

Con base en las CDC se tomó el 50\% de frecuencia y se restó el caudal ecológico, determinando así la oferta total, presentada en la Tabla 4.

Figura 5. CDC Estación Puente Calamar.

Tabla 4. Oferta del Río Frío

\begin{tabular}{|c|c|c|c|c|c|}
\hline Estación & $Q\left(m^{\wedge} 3 / s\right)$ & $\operatorname{Qmin}\left(m^{\wedge} \mathbf{3} / \mathbf{s}\right)$ & $\mathbf{Q e c o}\left(m^{\wedge} \mathbf{3} / \mathbf{s}\right)$ & $\operatorname{Oferta}\left(m^{\wedge} \mathbf{3} / \mathbf{s}\right)$ & Oferta (I/s) \\
\hline Pozo Hondo & 0,55 & 0,029 & 0,00725 & 0,5428 & 542,8 \\
\hline Páramo Guerrero & 0,0947 & 0,002 & 0,0005 & 0,0942 & 94,2 \\
\hline Puente Virginia & 1,246 & 0,001 & 0,00025 & 1,2458 & 1245,8 \\
\hline Puente Cacique & 1,48 & 0,013 & 0,00325 & 1,4768 & 1476,8 \\
\hline Puente Calamar & 0,936 & 0,029 & 0,00725 & 0,9288 & 928,8 \\
\hline
\end{tabular}

La figura 6 presenta el mapa resultante de la oferta (Ver anexo). se presenta el mapa resultante de la oferta.

\section{Objetivo dos. Determinación de la de-} manda superficial en los municipios pertenecientes a la subcuenca hidrográfica del Río Frío en Cundinamarca. Para la determinación de la demanda industrial y pecuaria, se manejó la información suministrada por la Corporación Autónoma regional de Cundinamarca (CAR) de las Concesiones de Agua del Censo de 2009, teniendo en cuenta los módulos de consumo correspondientes a cada industria y cabezas de ganado.

Por otro lado, para el cálculo de la demanda doméstica se consideró la proyección poblacional del DANE para el año 2017 y las áreas de cada municipio correspondiente a la subcuenca del Río Frío, y por último, para establecer la demanda agrícola, se hizo uso del shapefile de cobertura de uso del suelo del IGAC y los módulos de consumo por cultivo para el Río Frío.Los módulos de consumo fueron tomados del Estudio de Módulos de Consumo de la CAR (2005), siendo la Demanda Hídrica Total la suma de todas las anteriores.

En la Tabla 5 y en la Figura 2 se muestran los resultados obtenidos.

Tabla 5. Demanda de Río Frío

\begin{tabular}{l|c} 
Demanda & Caudal (L/s) \\
\hline Doméstica & 56,110 \\
\hline Industrial & 3,130 \\
\hline Pecuaria & 0,215 \\
\hline Agrícola & 2392,870 \\
\hline Total & $\mathbf{2 4 5 2 , 3 2 5}$ \\
\hline
\end{tabular}




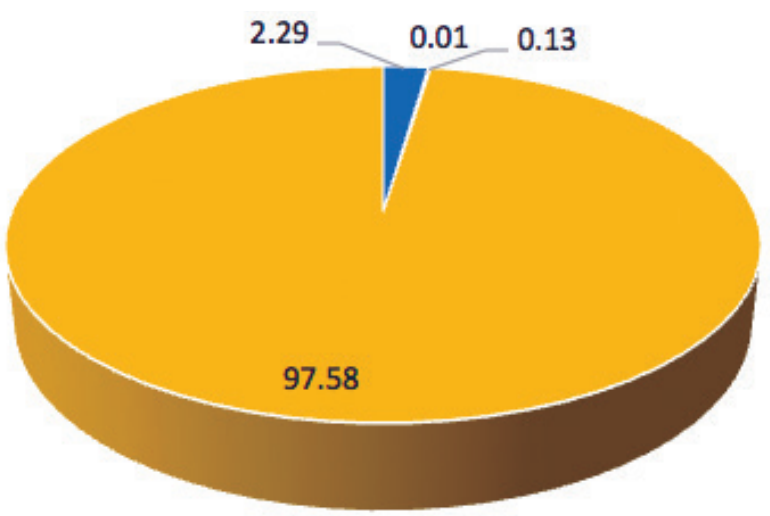

- Doméstica - Industrial = Pecuaria = Agrícola

Figura 7. Porcentaje de demanda del Río Frío.

Teniendo en cuenta que el $97,58 \%$ de la demanda es de origen agrícola, se presenta el mapa resultante de Demanda Agrícola en la figura 8 (Ver anexo).

Objetivo tres. Relación oferta-demanda. En la Tabla 6 se observan los resultados del balance y del Índice de Escasez obtenidos.

Tabla 6. Resultados del balance y del Índice de Escasez

\begin{tabular}{l|c}
\hline Demanda Total (I/s) & $\mathbf{2 4 5 2 , 3 2 5}$ \\
\hline Oferta (I/s) & 1476,8 \\
\hline Balance (I/s) & $-975,525$ \\
\hline Índice de Escasez & 166 \\
\hline Categoría & Demanda Alta \\
\hline
\end{tabular}

Objetivo cuatro. Evaluación de la calidad del agua de la subcuenca hidrográfica del Río Frío. Para realizar la evaluación del ICA mencionado anteriormente, se tuvieron en cuenta los valores de diversas variables físicas y químicas proporcionadas por la autoridad ambiental, CAR Cundinamarca, en 4 puntos de muestreo a lo largo de la subcuenca media y baja del Río Frío. Dichos puntos de muestreo fueron considerados como significativos debido a que en estas divisiones de la cuenca se presentan impactos negativos sobre el recurso hídrico; ya que, aledañas a estas subcuencas, se encuentran las poblaciones y las diversas industrias que agotan dicho recurso y generan vertimientos al río, contaminándolo y deteriorando la calidad de éste. En la Tabla 7 se presentan los 4 puntos de muestreo elegidos.

Tabla 7. Puntos de muestreo para determinar la calidad del agua del Río Frío.

\begin{tabular}{|c|c|c|c|}
\hline $\begin{array}{l}\text { Punto } \\
\text { de muestreo }\end{array}$ & $\mathbf{N}$ & $\mathbf{E}$ & Cuenca \\
\hline $\begin{array}{l}\text { Río Frío aguas } \\
\text { abajo de la } \\
\text { empresa Rosas } \\
\text { de Colombia }\end{array}$ & 1037616 & 998700 & Media \\
\hline $\begin{array}{l}\text { Estación } \\
\text { Puente } \\
\text { Virginia }\end{array}$ & 1036500 & 1001200 & Baja \\
\hline $\begin{array}{l}\text { Estación } \\
\text { Puente } \\
\text { Cacique }\end{array}$ & 1029550 & 1001330 & Baja \\
\hline $\begin{array}{l}\text { Río Bogotá aguas } \\
\text { abajo del Río Frío }\end{array}$ & 1026531 & 998628 & Baja \\
\hline
\end{tabular}

De este modo, para el cálculo de este índice se tuvieron en cuenta también las mediciones de las variables $\mathrm{pH}$, demanda química de oxígeno, sólidos suspendidos totales, conductividad eléctrica y oxígeno disuelto en los 4 puntos de muestreo presentados anteriormente, durante el segundo semestre del año 2014. Los datos recogidos se detallan en la Tabla 8. 
Tabla 8. Valores de los parámetros físicos y químicos de los puntos de muestreo del Río Frío utilizados para el cálculo del ICA)

\begin{tabular}{|c|c|c|c|c|c|}
\hline $\begin{array}{l}\text { Punto de } \\
\text { muestreo }\end{array}$ & $\begin{array}{l}\text { Oxígeno } \\
\text { disuelto } \\
\left.\text { ( } \mathrm{mgO}^{2} / \mathrm{L}\right)\end{array}$ & $\begin{array}{l}\text { Demanda química } \\
\text { de oxígeno } \\
\left(\mathrm{mgO}^{2} / \mathrm{L}\right)\end{array}$ & $\begin{array}{c}\text { Sólidos } \\
\text { suspendidos } \\
\text { totales }(\mathrm{mgSST} / \mathrm{L})\end{array}$ & pH & $\begin{array}{l}\text { Conductividad } \\
(\mathrm{mS} / \mathrm{cm})\end{array}$ \\
\hline $\begin{array}{l}\text { Río Frío aguas abajo } \\
\text { de la empresa Rosas } \\
\text { de Colombia }\end{array}$ & 6,40 & 11,00 & 10,00 & 6,40 & 54,90 \\
\hline $\begin{array}{l}\text { Estación Puente } \\
\text { Virginia }\end{array}$ & 7,10 & 11,00 & 5,00 & 7,40 & 51,70 \\
\hline $\begin{array}{l}\text { Estación Puente } \\
\text { Cacique }\end{array}$ & 0,60 & 168,00 & 58,00 & 7,20 & 292,00 \\
\hline $\begin{array}{l}\text { Río Bogotá aguas } \\
\text { abajo del Río Frío }\end{array}$ & 0,90 & 42,40 & 19,00 & 7,00 & 275,00 \\
\hline
\end{tabular}

Con los anteriores datos y utilizando la respectiva ecuación (3) se procedió a calcular el valor del ICA para cada punto de muestreo presentado en la Tabla 9.

Tabla 9. Valores del ICA para cada punto de muestreo

\begin{tabular}{l|c|c} 
Punto de muestreo & ICA & Calificación de la calidad del agua \\
\hline $\begin{array}{l}\text { Río Frío aguas debajo de la empresa } \\
\text { Rosas de Colombia }\end{array}$ & 0,84 & Aceptable \\
\hline Estación Puente Virginia & 0,91 & Buena \\
\hline Estación Puente Cacique & 0,54 & Regular \\
\hline Río Bogotá aguas abajo del Río Frío & 0,46 & Mala \\
\hline
\end{tabular}

Con base en los resultados anteriores, se puede visualizar el estado del Río Frío mediante la figura 9 (Ver anexo).

\section{Discusión}

La cuenca del Río Frio se encuentra habitada en gran parte por una población de índole rural, con excepción del casco urbano de Chía, el cual cubre su demanda con otras fuentes de abastecimiento como es el Proyecto TibitocCasablanca.Teniendo en cuenta lo anterior y que los municipios que conforman la cuenca basan su economía en la ganadería y la agricultura, la demanda del Río Frío se basa en el riego de pastos y cultivos, considerando que es una zona de baja precipitación.

Por otro lado, en comparación con el POMCA del Río Frío realizado en el año 2006, en el cual se obtuvo una demanda total de $1320 \mathrm{~L} / \mathrm{s}$ y una oferta de $1470 \mathrm{~L} / \mathrm{s}$, se puede observar que la oferta del río, al transcurrir los años, no ha presentado una variación representativa con respecto a la demanda, la cual aumentó aproximadamente 1000 L/s en 11 años, lo cual se puede deber al crecimiento poblacional e industrial que se ha presentado en los últimos años en esos municipios debido a la conurbación de Bogotá. 
Así mismo se puede observar una variación en el Índice de Escasez, en donde para el POMCA del año 2006, el resultado fue de 88,4\% siendo categoría alta; donde, en comparación con el presente estudio se puede observar que aumentó un $77,6 \%$, sin presentar variación en la categoría ya que en los dos casos el porcentaje es mayor a 40.

En cuanto a la calificación del estado de la calidad del agua del río Frío, mediante la determinación del Índice de Calidad del Agua en cada punto de muestreo, se identificaron cuatro calificaciones de las cinco establecidas por el IDEAM, correspondientes a "Buena" para la estación Puente Virginia, y para la subcuenca alta del Río Frío debido a que sobre esta parte alta de la cuenca y en este punto no confluyen vertimientos de aguas residuales o industriales provenientes de poblaciones o actividades económicas, reflejado esto en valores mínimos de las variables que inciden en la determinación del ICA. También se identificó "Aceptable" para el punto aguas abajo de la empresa Rosas de Colombia, una calidad menor que la mencionada anteriormente; posiblemente, debido a los vertimientos que genera esta empresa sobre el río. Igualmente, se estableció la calificación "Regular" para la estación Puente Cacique, y "Mala" para el punto aguas abajo del Río Frío, lo cual puede ser debido a la ubicación de estos puntos de muestreo, ya que al ser los puntos más bajos de la subcuenca, son los que mayor contaminación reciben debido a las aguas residuales e industriales provenientes de poblaciones y actividades económicas aledañas. En este sentido, en estos puntos de muestreo la composición química del agua, así como la fauna y la flora, se ven afectadas debido a la degradación de este recurso hídrico, disminuyendo su calidad y, por lo tanto, haciendo este recurso poco apto para suplir las necesidades de las poblaciones que requieren de éste para diversas actividades económicas.

\section{Conclusión}

Al realizar la evaluación de la subcuenca del Río Frío, se determinó que la oferta hídrica suministrada por el cuerpo de agua no logra satisfacer la demanda superficial de los municipios aledaños utilizada para la realización de sus actividades económicas; principalmente, el riego de pastos y cultivos de papa, arveja, zanahoria y maíz; generando una fuerte presión sobre este recurso hídrico.

En cuanto a la calidad del agua del Río Frío, se observó que presenta una "buena" calidad en la subcuenca alta, indicando que el recurso hídrico de esta zona es adecuado para el desarrollo de las diferentes actividades económicas de los municipios,; sin embargo, se obtuvo también que la calidad de este cuerpo hídrico tiende a disminuir en las subcuencas media y baja al reflejar estados de calidad "aceptable", "regular" y "malo" , respectivamente, en el transcurso del río; por lo cual se obtiene que el recurso en general, presenta indicios de una contaminación significativa, y por ende, se considera que el Río Frío no es apto para suplir las diversas necesidades de los municipios, siendo necesario la implementación de prácticas de producción más limpias por parte de los sectores productivos para mitigar la generación de residuos que causen impactos negativos sobre el Río Frío.

\section{Literatura citada}

Corporación autónoma regional de cundinamarca - CAR, (2005). ACUERDO No. 31 del 19 de septiembre de 2005". Recuperado de: https://www. indumil.gov.co/wp-content/uploads/2016/03/2.Acuerdo-CAR-31-de-2005.pdf

Corporación autónoma regional de cundinamarca - CAR, (2006). plan de ordenación y manejo de la cuenca hidrográfica del río bogotá". Recuperado de: http://www.alcaldiabogota.gov. 
co/sisjur/adminverblobawa?tabla $=T$ NORMA ARCHIVO\&p_NORMFIL_ID $=305 \& f_{-}$NORMFIL $F I L E=X \&$ inputfileext $=$ NORMFIL_FILENAME

Corporación autónoma regional de cundinamarca CAR, (2009). Resolución opsc nº 00026 de 2009. Recuperado de: http: //www.alcaldiabogota.gov. co/sisjur/normas/Norma1.jsp?i=36216.

Flores, M. Gestión integrada de los recursos hídricos, de la cuenca hidrológica del río papagayo, estado de guerrero. Trabajo de investigación (Doctor en Ciencias y Tecnología del Agua). Instituto Mexicano de Tecnología del Agua. Facultad de ingeniería. México, 2014, p. 270.

Instituto de Hidrología, Meteorología y Estudios Ambientales - IDEAM, (2004). Metodología para el cálculo del índice de escasez de agua superficial". Recuperado de: http://documentacion. ideam.gov.co/openbiblio/bvirtual/021143/metodologia-calculo.pdf.

Instituto de Hidrología, Meteorología y Estudios Ambientales - IDEAM, (2013). Índice de calidad del agua en corrientes superficiales (ICA). Recuperado de: http://www.ideam.gov.co/documents/24155/125494/36-3.21_HM_Indice_ calidad_agua_3_FI.pdf/9d28de9c-8b53-470e82ab-daca2d0b0031.
Instituto de Hidrología, Meteorología y Estudios Ambientales - IDEAM, (2014). Estudio Nacional del Agua". Recuperado de: Available: http:// documentacion.ideam.gov.co/openbiblio/bvirtual/023080/ENA_2014.pdf.

Puyol, A. F. (2006). Principios y fundamentos de la hidrología superficial. Mexico : Universidad Autonoma de Mexico . Obtenido de http:// www.uamenlinea.uam.mx/materiales/licenciatura/hidrologia/principios_fundamentos/libroPFHS-05.pdf

Organización de las Naciones Unidas para la Educación, la Ciencia y la Cultura - UNESCO, (2006). Water a shared responsibility The United Nations World Water Development Report 2. Recuperado de: http://unesdoc.unesco.org/ images/0014/001444/144409E.pdf.

Venegas, C. (2013). Estudio preliminar de la subcuenca del río frío en el tramo comprendido por el municipio de cajicá". Recuperado de: http://repository.unimilitar.edu.co/bitstream/10654/11079/1/ Entrega\%20Final_Estudio\%20Preliminar \%20Estado\%20del\%20R\%C3\%ADo\%20Fr\%C3\%ADo_ Cesar\%20Venegas.pdf. 